\title{
Traumlogiken des Krieges \\ Alexander Lernet-Holenia und der Erste Weltkrieg in der Habsburger Monarchie
}

\section{1.}

Man mag Erich Maria Remarques berühmten Romantitel Im Westen nichts Neues, zumal in der Zeit nach dem Centenaire-Gedenken an den Ausbruch des Ersten Weltkriegs, auch auf die Forschung übertragen. In der Vielzahl auch literatur-, medien- und kulturgeschichtlicher Forschungen zum Ersten Weltkrieg ${ }^{1}$ werden bestimmte Thematisierungsmuster, Schwerpunktsetzungen, ja sogar eine spezifische Vorstellung von dem deutlich, was es heißt, vom Ersten Weltkrieg zu erzählen oder ihn künstlerisch darzustellen. ${ }^{2}$ Je umfassender das Bild wird, umso mehr entsteht doch auch der Verdacht, dass das, was mit solchen Forschungen zutage gefördert wird, eben nur ein bestimmtes und sehr eng gerahmtes Bild ist. Nimmt man das Bild für dasjenige, was es abbildet, läuft man Gefahr, es in einen Mythos (im Sinne von R. Barthes) zu verwandeln, der sich auf bestimmte Orte, Ereignisse und Texte einschränken lässt.

1 Beispielhaft sei herausgegriffen: Werber/Kaufmann/Koch (Hgg.): Erster Weltkrieg.

2 Z.B. Hüppauf: Kriegsliteratur.
In dem Erzählwerk von Alexander Lernet-Holenia (1897-1976) wird der Erste Weltkrieg aus einer besonderen Perspektive auf das österreichischungarische Kaiserreich mit einem geographischen Schwerpunkt auf dem Balkan geschildert. Für den Blick auf diesen Raum ist Lernet-Holenia deswegen so bedeutsam, weil er Geopolitik, eine ausgeklügelte Erzähltechnik und eine spezifische Modernität der Literatur verbindet. Der Beitrag zeigt an der Novelle Der Baron Bagge (1936) und am Roman Die Standarte (1934), wie der Autor ein Modell von Kriegsliteratur entfaltet, das als Traumlogik des Krieges beschrieben wird und mit dem er bspw. die prekäre Frage nach der postimperialen Identität Österreichs aufgreifen kann. 
In der Tat ist der Eindruck nicht von der Hand zu weisen: Der Krieg im Westen, in Frankreich, der Stellungskrieg mit all seinen Auswüchsen, Städte- oder Schlachtnamen wie Verdun oder Somme, der gescheiterte Schlieffenplan, aber auch der Gaseinsatz, der Einsatz neuer Techniken (tanks/Panzer), all diese Aspekte sind zu einem Paradigma des Ersten Weltkriegs geworden, das die Perspektive doch sehr stark einengt und zu einer Schieflage in der Wahrnehmung der geopolitischen Situation jener Zeit führen kann. ${ }^{3}$ Der heutige Blick auf den Ersten Weltkrieg geht geographisch vorrangig (natürlich nicht ausschließlich) ${ }^{4}$ nach Westen, vielleicht mit einigem Recht, kamen doch mit den genannten Aspekten jene Momente zum Tragen, die diesen Ersten Weltkrieg wesentlich charakterisieren - und auch mit entschieden haben. Das gilt für die politische Geschichte wie für die Literatur- und Mediengeschichte. Insofern nimmt es nicht wunder, dass auch die Autoren (z.B. Remarque, Döblin, Köppen) und ihre entsprechenden und z.T. hochrangig kanonisierten literarischen und künstlerischen Texte sich vorrangig um den Krieg im Westen kümmern. Für die deutsche (nicht: deutschsprachige) Literatur zumal war der Krieg im Osten von >marginaler` Bedeutung, der Krieg Österreich-Ungarns, der in Norditalien, auf dem Balkan und im Südosten des europäischen Kontinents stattfand, spielte für deutsche Autoren kaum eine nennenswerte Rolle. Wenn aber das Bild vom Ersten Weltkrieg durch eine solche Perspektive geprägt ist, so muss eine veränderte Perspektive auch ein verändertes Bild zutage fördern. Wenn man zudem annimmt, dass der Blick nach Westen auch bestimmte Darstellungs- und Thematisierungsweisen mit sich gebracht hat, so muss der andere geographische, historische, politische und kulturelle Fokus, nämlich auf die Habsburger Monarchie und ihren Zusammenbruch, zu einer differenzierteren Sicht des Ersten Weltkriegs führen.

\section{2.}

Ändert man allerdings die geographische und geopolitische Blickrichtung und fokussiert auch in der Literatur den Ersten Weltkrieg aus einer Perspektive der Habsburger Monarchie mit dem Schwerpunkt auf den slawischen und insbesondere süd-slawischen Raum, so kann ein Autor nicht umgangen

3 Zur Geopolitik der Literatur vgl. Werber: Die Geopolitik der Politik und neuerdings ders.: Geopolitik zur Einführung; zu Österreich-Ungarn speziell ders.: Geopolitik.

4 Als Beispiel für eine andere Blickrichtung sei genannt: Angelow (Hg.): Der Erste Weltkrieg auf dem Balkan. Als Grundlagenwerk gilt: Rauchensteiner: Der Erste Weltkrieg. 
werden, der in diesem Krieg gekämpft hat, der Rittmeister war und in dessen erzählerischem Werk sich dieser Krieg aus dieser Perspektive facettenreich niedergeschlagen hat - und zwar auf verblüffende Weise auch mit jenem geopolitischen Schwerpunkt, wie er durch die angesprochene veränderte Blickrichtung mitgegeben ist. Die Rede ist von Alexander Lernet-Holenia (1897-1976). Wer also lesen will, wie die Habsburger Monarchie militärisch zusammengebrochen ist, wie sich das Heer insbesondere an den Frontlinien des Ostens und des Balkans aufgelöst hat, wie sich neue Nationalitäten und entlang dieser Entwicklungen neue, insbesondere kulturelle Verwerfungslinien gebildet haben, aber auch, wie sich dieser Krieg und die Niederlage der Habsburger Monarchie auch mentalitätsgeschichtlich ausgewirkt und niedergeschlagen haben, wie die dramatischen historischen Erfahrungen zu einer neuen postimperialen österreichischen Identität geführt haben oder eine solche überhaupt erst problematisch haben werden lassen und wie schließlich Krieg und Niederlage die Psyche von Menschen z.T. traumatisch bestimmt haben, ${ }^{5}$ der muss in die Romane und Erzählungen LernetHolenias blicken. Die Rekonstruktion seiner literarischen Gestaltung der genannten Prozesse wäre - so die Hypothese - ein Beitrag zur Diskussion über die veränderte Blickrichtung auf den Ersten Weltkrieg.

\section{3.}

Dass man in Lernet-Holenias Werk einen der profiliertesten Erzähler der Literatur des 20. Jahrhunderts erkennen kann, den Hilde Spiel, seine langjährige Mitarbeiterin beim österreichischen P.E.N.-Club, zu Recht mit Kleist (Lernet-Holenia hatte 1926 den Kleist-Preis verliehen bekommen) ${ }^{6}$ vergleicht, mag ja noch als Hinweis auf einen neu zu entdeckenden Geheimtipp der Literaturgeschichte durchgehen. ${ }^{7}$ Darüber hinaus kann man Verblüffendes erfahren, zum Beispiel, dass seine Biografie sich liest als hätte er sie selbst als Roman verfasst, in der all das vorkommt, was seine Militärromane ausmacht, eine Liebesnacht etwa, ein Offizier, geheimnisvolle Befehle, seltsames Verhalten, dunkle Geheimnisse und die Lebensgeschichte eines jungen Soldaten, der zu schreiben beginnt.

5 Dassanovsky: Phantom Empires.

6 Dass Hans Joachim Kreutzer (Der Kleist-Preis 1912-1932-1985, S. 12) die Verleihung »heute [1986 ] nicht überzeugend « findet, ist nun selbst wiederum eine historische Fehleinschätzung.

7 Spiel: Welche Welt ist meine Welt?, S. 221; dies.: Lernet-Holenia, Alexander. 
Den adeligen Mädchennamen seiner Mutter Holenia darf er erst später ab einer Adoption im Jahre 1920 führen; zunächst heißt er Lernet wie sein Vater, der gar nicht sein Vater war. Man kennt die Gerüchte, denen Roman Rocek in seiner Biografie Die neun Leben des Alexander Lernet-Holenia nachgegangen ist. ${ }^{8}$ Er hat den Indizienbeweis geführt, dass der Marineoffizier Lernet dazu herhalten musste, einen Fehltritt von Lernet-Holenias Mutter, dem der Dichter entsprang, zu vertuschen. Der wirkliche Vater hatte wohl allerhöchstes Blut in seinen Adern und war vermutlich einer der Habsburgischen Erzherzöge. So hieß es später, Lernet-Holenia sei der letzte Habsburger gewesen, dem es vergönnt war, in der Hofburg bis zu seinem Tode zu leben.

Man könnte das Thema der unsicheren Identität geradezu als ein Leitmotiv aller seiner Romane bezeichnen. Doch man muss nicht in einen naiven Biografismus verfallen, um festhalten zu können, dass seine Biografie als Folie seiner Sujetgestaltung relevant wird; bei Robert Dassanowsky heißt es entsprechend: »Alexander Lernet-Holenia’s inability to define his own persona is a revealing reflection of his identification with an Austria haunted by a phantom Empire and of his struggle to define the quality what is Austrian. $\ll^{9}$

Das muss nicht verwundern, ist doch Alexander Lernet-Holenias Lebensweg auch von der österreichischen Geschichte des 20. Jahrhunderts geprägt. Er wird 1897 in Wien geboren, ist von 1916 bis 1918 Soldat in der k.u.k. Armee, vermutlich als Rittmeister oder in einer ähnlich equestrischen Position und Funktion, er beginnt Gedichte zu schreiben, schlägt nach dem Krieg die Laufbahn als freier Schriftsteller ein, in der er reüssiert; er nimmt kurz auf deutscher Seite am Zweiten Weltkrieg teil; er wird verwundet und wird danach Mitarbeiter der Heeresfilmstelle. 1969 wird er Präsident des österreichischen P.E.N.-Club. Von diesem Posten trat er nach mancherlei Drohungen 1972 zurück, aus Protest gegen die Verleihung des Literaturnobelpreises an Heinrich Böll. Dass dies ein Vorwand war, der andere Gründe und Zerwürfnisse verdecken sollte, steht zu vermuten. Wer allerdings die Konstellation als ästhetischen Konflikt zweier Erzähler deuten mag, findet in der Bölls Erzählweise weit überragenden Technik Lernet-Holenias zahlreiche Plausibilitätsgründe. Zuletzt lebt er in einer Wohnung der Wiener Hofburg. Er stirbt in Wien 1976.

Lernet-Holenias Werk hat drei Säulen: seine überwiegend frühe Lyrik, seine mittleren Theaterarbeiten und seine späteren Romane und Erzäh- 
lungen. Natürlich überschneiden sich die Grenzen und Phasen. Zudem verschmolz sein dramatisches und sein episches Schreiben in Arbeiten für den Film, denn Lernet-Holenia hatte ein untrügliches Gespür für gute, spannende Geschichten und ihre dramaturgische Umsetzung, nicht nur episch in Roman und Erzählung, sondern eben auch dramatisch und performativ für das Theater und den Film. Dennoch, die lyrische Arbeit war ihm immer Paradigma seines Schreibens.

Er hat früh einen normativen Literaturbegriff entwickelt, der eigentlich Literatur nur in gebundener Sprache verwirklicht sah und den er in seinem lyrischen Werk umsetzen wollte. ${ }^{10}$ Aus diesem Grund hat er selbst seine späteren Romane und Filmarbeiten nur als Mittel zum Geldverdienen abgetan. Dass er sich dabei selbst Unrecht getan hat, hat nicht nur Hilde Spiel herausgestellt. Man kann sogar sagen, dass diese Selbstverkennung - »not taking his own work seriously enough $\aleph^{11}$ - gerade die Voraussetzung für eine epische, fiktionale und narrative Freiheit war, um jene Sujets zu gestalten, die seiner eigenen Identitätsproblematik näher kamen als mancher andere Stoff. Aus einer literaturgeschichtlichen Perspektive kann man erkennen, dass er mit seiner Epik einen wesentlichen Beitrag zu einer ästhetischen Aufarbeitung von grundlegenden und neuen Erfahrungen geliefert hat, die der Erste Weltkrieg mit sich gebracht hat - zumal für die Habsburger Monarchie.

\section{4.}

Für den vorliegenden Zusammenhang mag von besonderem Interesse sein, dass in Lernet-Holenias Erzählwerk ein geopolitischer Fokus, eine ausgeklügelte Erzähltechnik und eine spezifische Modernität der Literatur einen Funktionszusammenhang bilden. Der Autor operiert mit kunstvoll gestaffelter Wissensvergabe, die über lange Passagen Unsicherheit erzeugt und erhält, was insbesondere in kriminalistischen Sujets sinnfällig wird. Dabei wird die Frage nach der persönlichen und der kollektiven Identität einer Gesellschaft (oder eines Volkes im Sinne der zerbrechenden Vielvölkergemeinschaft der Habsburger Monarchie) vor allem mit der Frage nach der Wahrnehmung von Zeit und Raum verknüpft, so dass die Wahrnehmungsstrukturen die Strukturen der Narration determinieren, was als ein Kennzeichnen moderner Literatur seit der Jahrhundertwende gelten darf. 
Im Zentrum steht dabei eine unsichere Identität, die in den entscheidenden Phasen der Konfliktgestaltung einen einheitlichen Erfahrungsraum ebenso wie eine stringente Biografie nicht mehr garantieren kann.

Wahrnehmung und Narration dürfen bei Lernet-Holenia aber nicht gleichgesetzt werden. Denn man muss deutlich machen, dass er andere literarische Entwicklungen der deutschsprachigen Literatur, wie sie z.B. (allerdings mit einem Schwerpunkt vor allem auf der Literatur der Weimarer Republik) mit dem Schlagwort von der Krise des Romans bezeichnet werden, gerade nicht mitmacht. ${ }^{12}$ Das ist der wichtigste Punkt, auf den man die pauschale Feststellung von Robert Dassanowsky zuspitzen kann, bei Lernet-Holenia könne man »avoiding the literary trends of the interwar and postwar era « feststellen. ${ }^{13}$ Wahrnehmungskrisen führen bei diesem Autor nicht zu Erzählkrisen, im Gegenteil: die krisenhaften Wahrnehmungen liefern gerade das produktive Sujet seines Erzählens. Das erzählerische Werk liefert ein Spektrum wohl kalkulierter Erzähltechniken mit selbstreflexiver Dimension, wobei sich die Biografie des Autors, die Erzähltechnik und die Reflexion des Erzählens wechselseitig erhellen.

Nun scheint es sich gerade anzubieten, Lernet-Holenias Texte als Beispiele für jenes Modell zu lesen, das Claudio Magris in seiner Dissertation aus dem Jahr 1963 für die Literatur des österreichischen Kulturraums entworfen und dem er den Namen eines >Habsburgischen Mythos` gegeben hat. ${ }^{14}$ Zunächst mag dies eine faszinierende Lektürelinie vorgeben, wird doch die Nachkriegserfahrung bei Lernet-Holenia immer als Erfahrung des Verlustes und des Unwiederbringlichen geschildert, so z.B. in dem hierfür zentralen Roman Beide Sizilien aus dem Jahr 1942, der eine Konstituente des Herrschaftsmythos der Habsburger ebenso im Titel trägt wie den Namen eines berühmten Regiments, dessen Name seinerseits auf diesen Herrschaftsmythos verweist. In diesem Roman wird eine Gruppe ehemaliger Offiziere, nachdem sich ihr Zusammenhalt längst aufgelöst hatte, noch einmal zusammengeführt, weil ihre Mitglieder nach und nach ermordet werden oder unter seltsamen Umständen zu Tode kommen - durch einen Mörder, der permanent die Identität wechselt.

Magris wird Lernet-Holenia jedoch eigentümlicherweise weder literaturkritisch noch konzeptionell gerecht. Er behandelt diesen Autor - vielsagend - im Kapitel »Die unbedeutendere Literatur«, die er so bilanziert: »Von dieser eher oberflächlichen Strömung kann man gewiß nicht viel 
erwarten.« ${ }^{15}$ Dass er Lernet-Holenia als »lebendigsten Schriftsteller« davon etwas ausnimmt, ändert jedoch nichts daran, dass er seinen wichtigsten und bekanntesten Roman, einen Militärroman, Die Standarte aus dem Jahr 1934, der den »Zusammenbruch der Monarchie» schildere, kaum angemessen einschätzt:

Diesem Werk fehlt es nicht an Lebendigkeit, Eindringlichkeit und einem Sinn für schicksalhafte Tragik. Das Ende des Reiches wird sowohl in seinen äußeren Aspekten - die militärische Niederlage, mehr noch aber das Zerbröckeln der moralischen Einheit des Heeres, an deren Stelle nationale Ressentiments treten - als auch an inneren Erscheinungen verdeutlicht, nämlich der geistigen Müdigkeit, die selbst die tapfersten Verteidiger der Monarchie entkräftet. ${ }^{16}$

Diese Einschätzung greift mit ihrem zu engen Blickwinkel, der nahezu ausschließlich die >äußeren Aspekte` hervorhebt, das erzählerische Moment aber nicht beachtet, zu kurz. Besonders gilt das, wenn man Lernet-Holenias Art und Weise zu erzählen mit anderen Modellen konfrontiert. Man müsste umgekehrt verfahren und mit Lernet-Holenia der Magris'schen Konzeption mehr Tiefenschärfe verleihen, indem man zeigt, wie dies Robert Dassanowsky getan hat, dass es hier nicht um die Restitution einer Idee in der Form eines von der Literatur inszenierten Mythos geht, sondern vielmehr um die Bearbeitung historischer Folgelasten, die sich in der prekären nationalen Identität Österreichs und der Definition des Österreichischen niederschlägt. ${ }^{17}$

Vielleicht ließe sich Lernet-Holenias Position ohnehin besser konturieren, wenn man sie nicht in den Habsburger-Mythos, sondern in das Feld der zeitgenössischen deutschsprachigen Literatur einfügt. Es gibt beispielsweise einige biografische Parallelen zu Ernst Jünger, die auch literaturgeschichtlich interessant werden können. Die Geburtsdaten der beiden Dichter liegen nur zwei Jahre auseinander, beide sind Soldaten im Ersten Weltkrieg, beide beginnen danach ihre Schriftstellerkarriere (die auf Erfahrungen im Krieg zurückgehen), beiden ist das soldatische Wesen, das sie zu einer Frage der Einstellung hochstilisieren, Lebensprinzip, beide nehmen - auf deutscher Seite - am Zweiten Weltkrieg teil und sind - auch durch ihre neueren Publikationen - den Machthabern suspekt. Und doch hat Ernst Jünger eine ganz andere Art gefunden, über den Krieg zu schreiben, was deutlich wird, wenn man auf die ersten Fassungen der Stahlgewitter seit $1920^{18}$ oder auf das vorausgehende, von Helmuth Kiesel herausgegebene 
Kriegstagebuch blickt. ${ }^{19}$ Man kann den Kontext noch ein wenig ausweiten: So unterschiedlich Jünger einerseits und beispielsweise Erich Maria Remarque (mit dem Roman Im Westen nichts Neues, 1928) oder Edlef Köppen (mit dem Roman Heeresbericht, 1930) auf der anderen Seite auch sein mögen, sie alle nehmen einen Gegenpart zu Lernet-Holenia ein. Dies ist auch ein Unterschied, der durch den geopolitischen Fokus gegeben ist. Auch in diesem Vergleich wird deutlich: Ein anderer Fokus führt zu veränderten Wahrnehmungen des Krieges und zu anderen Formen des Erzählens. Geht es bei den genannten deutschen Autoren um die Sinnlosigkeit des Krieges in der Drastik seiner Schilderung, so geht es bei Lernet-Holenia um die psychosozialen Folgelasten des verlorenen Krieges, was andere Formen der Darstellung mit sich bringen muss.

\section{5.}

Ja, man könnte das Spektrum noch stärker und international ausweiten und nach paradigmatischen Modellen der Kriegsliteratur, wie dies Bernd Hüppauf skizziert hat, ${ }^{20}$ oder gar des Writing War fragen, wie dies zuletzt Daniela Kirschstein in einer imposanten Monographie unter diesem Titel vorgeführt hat. ${ }^{21}$ Bernd Hüppauf hat mit seinem Überblicksartikel zur Kriegsliteratur zum Ersten Weltkrieg deutlich gemacht, dass die »Beziehung zwischen Literatur, Kunst und modernem Krieg konstitutiv ist «. Daraus zieht Hüppauf literaturtheoretische Konsequenzen: »Die Interpretation von Kriegsliteratur muss von dieser inneren Beziehung ausgehen und verfehlt ihre Frage, sobald sie Krieg als ein bloßes Motiv oder einen Gegenstand von Literatur und Kunst auffasst. « $^{22}$ Vielmehr gelte es, ihm zufolge, zwischen den Positionen einer mimetischen Abbildung, Repräsentation oder Dokumentation, eines authentischen Wahrheitsanspruchs und einer Mythologisierung des Krieges vor allem auf jene ästhetischen Positionen der Moderne zu achten, die sich erst mit der Kriegsliteratur ausbilden konnten. Daniela Kirschstein geht über diese Perspektivierung noch hinaus, indem sie das Schreiben über den Krieg als Ethnographie deutet. Sie öffnet damit überhaupt erst den Blick für paradigmatische Formen des Schreibens über den Krieg, weil sie dafür ein vorausliegendes, konstitutives Muster identifiziert. Dass auch bei einem 
Autor wie Lernet-Holenia der ethnographische Blick Wesentliches zutage fördern könnte, steht bei der Leistungsfähigkeit dieses Modells sowohl auf der Objektebene des literarischen Textes als auch auf der Metaebene einer literaturwissenschaftlichen Analyse außer Frage, doch ich möchte im Falle Lernet-Holenias noch einen Schritt weitergehen, weil bei Lernet-Holenia sich ein Modell abzeichnet, das über die Ethnografie hinausgeht.

Denn das ist das Besondere, das sich schon in einer ersten Lektüre aller späteren Romane, insbesondere der von Lernet-Holenia selbst so genannten Militärromane, unmittelbar erschließt. Man könnte sagen, dass alle diese Romane von einer romantischen Struktur der Sujetgestaltung leben, die in dem besagten komplexen Verhaltnis zur Biografie des Autors steht. Die Weisheit ’pater semper incertus« kann daher geradezu als poetisches Prinzip gelten. Alle Romane und Erzählungen operieren durchweg mit einem Konzept der unsicheren Identität der Hauptfigur. Die Bandbreite der Ausgestaltung ist groß, dennoch lässt sich ein struktureller Kern erkennen. Alle Hauptfiguren werden durch die Geschehnisse, in die sie geraten und die den Erzählimpuls abgeben, zutiefst irritiert; die Konstituenten der Wahrnehmung ihres Selbst und ihrer Welt werden erschüttert. Sie wissen nicht mehr, wer sie sind, noch wo sie sind. Sie gehen allesamt durch eine Passage extremer Unsicherheit. Sie wechseln, mehr oder weniger freiwillig ihre Identität (Ich war Jack Mortimer, 1933), ihr Geschlecht (Abenteuer eines jungen Herrn in Polen, 1931), bisweilen sogar ihren ontologischen Status vom Tod zum Leben (Die Auferstehung des Maltravers, 1936). Identitäten werden gestohlen oder von Toten übernommen (Ich war Jack Mortimer, 1933, Beide Sizilien, 1942, Graf Luna, 1955). Sie eilen unsicheren Liebesverhältnissen und geheimnisvollen Objekten ihres Begehrens (Mars im Widder, 1941, Baron Bagge, 1936, Mona Lisa, 1960), Phantomen (Graf Luna, 1955) und einfach ihrem Heil (Der Graf von Saint Germain, 1948) nach.

All diese Verunsicherungen sind vorderhand auf den historischen Kontext der erzählten Geschichten zurückzuführen, die allesamt in der Zeit nach dem Ersten Weltkrieg, nach der Habsburger Monarchie spielen und daher immer dort, wo sie einen Verlust und eine Unwiederbringlichkeit kompensieren, tatsächlich auf oberflächliche Art dem Habsburger-Mythos zuzurechnen wären. Aber darin erschöpfen sich die Texte nicht. Vielmehr sind die Sujets, die von der unsicheren Identität handeln, die Form der Narration und die Situierung der Texte in ihrem Entstehungskontext in der Zwischenkriegszeit auf engste miteinander korreliert; ja, mehr noch, die unsichere Identität ist jene Form, in der der Entstehungskontext und die Problempotenziale, die mit ihm gegeben sind, in den Texten selbst fiktionalisiert und erzählbar gemacht werden. 


\section{6.}

Um jedoch Lernet-Holenias Beitrag zu einem Modell des `writing war ‘ genauer bestimmen zu können, sollen zwei Texte ins Auge gefasst werden: die Novelle Der Baron Bagge aus dem Jahr 1936 und der Roman Die Standarte aus dem Jahr 1934. Die Erzählkonstruktion, die Lernet-Holenia in diesen beiden und in einer Vielzahl weiterer epischer Texte anwendet, mag man romantisch nennen, erlaubt doch ein realistischer Einstieg in die Erzählung die Erfahrung einer ontologisch unsicheren, potenziell phantastischen Realität. Die Anlage einer Rahmenerzählung lenkt von vornherein den Blick auf die Bedingungen des Erzählens von Geschichten und plausibilisiert somit die eigentümlichen narrativen Operationen, die die Texte dann inszenieren. Denn in beiden genannten Texten hat man es (auch) mit geradezu realistischen und sachlichen Darstellungen von Kriegsgeschehen in militärisch schwierigen oder aussichtlosen Situationen zu tun. Aber in beiden Texten tritt ein Ereignis ein, das die Kriegsdarstellung in den Hintergrund treten lässt, was es dem Leser wiederum erlaubt, den Krieg selbst aus einer anderen als der durch die Figuren vorgegebenen Perspektive zu sehen.

Baron Bagge, der Ich-Erzähler in der Binnengeschichte der gleichnamigen Novelle, findet auf einem Patrouillenritt, nach dem geheimnisvollen Übertritt über eine Brücke, alle seine Erwartungen plötzlich ins Gegenteil verkehrt: Sein selbstmörderisch draufgängerischer Vorgesetzter hat Glück, kein Feind ist sichtbar, kein Krieg wird spürbar, ein Dorf ist in festlicher Stimmung, ein junges Mädchen verführt ihn zum Sex, und sofort wird eine Hochzeit mit dem Vater des Mädchens vereinbart. Die Mutter des Mädchens, heißt es, sei vor langer Zeit gestorben. Später allerdings werden alle diese Erlebnisse als Traum entlarvt. Die Patrouille wurde in Wirklichkeit angegriffen und aufgerieben, nur Bagge hat im Koma überlebt. Traumwelt und wirkliche Welt sind geradezu direkt komplementär und somit zumindest negativ ineinander übersetzbar. Wer im Traum tot ist, lebt im Wachen und umgekehrt. Wer im Traum siegt, erlebt im Wachen seine Niederlage. So erfährt Baron Bagge, dass nur die Mutter des Mädchens noch lebt, das Mädchen und der Vater, die er im Traum kennengelernt hatte, seien im Kriegsgeschehen tatsächlich umgekommen.

Der Traum darf hier nicht vorschnell auf ein psychoanalytisches Motiv reduziert werden, vielmehr gilt es, die narratologische und die epistemologische Funktion des Traumes im Text zu erfassen. Tatsächlich ist der Traum ein motivisch konkretes Element einer grundlegenden Erzähllogik, die auch dort nachzuverfolgen ist, wo kein Traum geschildert wird. Der Traum ist vielmehr eine narratologische Operation, und die Traumerzählung kann 
somit als Erzähltechnik transparent gemacht werden. Der Traum nämlich erlaubt in konzentrierter Form, Wahrnehmungsstrukturen von Zeit und Raum und von zeitlich und räumlich fixierten Strukturen der Identität von Individuum und Gemeinschaft zu verflüssigen, aufzulösen und zu rekombinieren. Der Traum ist ein erzählerischer Kunstgriff, die erzählte Welt phantastisch erscheinen zu lassen - eine Funktionsbestimmung des Traums, die im Grunde genommen für alle phantastischen Sujets bei Lernet-Holenia gilt. Reinhard Lüth spricht hier von einer »Affinität von Erzähltechnik und Phantastik «, ${ }^{23}$ was vielleicht noch zu wenig gesagt ist, weil man tatsächlich von einer strukturell wechselseitigen Funktionalisierung und Instrumentalisierung ausgehen müsste. Die Umkehrung, die der Traum leistet, ist damit zugleich Erkenntnisinstrument, in seiner visuellen Funktionsweise vergleichbar mit dem Negativ einer Fotografie. So resümiert der Ich-Erzähler, Baron Bagge, am Ende der Novelle seine Erfahrung: »Denn in Wahrheit, so zuwider Phantastereien sonst mir sind, ist mir im Innersten der Traum noch Wirklichkeit und die Wirklichkeit eigentlich nur mehr wie ein Traum. $\ll^{24}$ Diese Selbstbeschreibung auf der Ebene der Figurenrede mag auch als poetologisches Prinzip auf der Textebene gelesen werden. Die Aussage bezieht sich außerdem auf die Verfremdung von Erfahrung, die sich zu einer komplementären Relation und mithin zu einer Negation gesteigert hat, und deswegen - ex negativo - ein Bild des Krieges und der Kriegserfahrung gibt. Dabei wird - und das ist das entscheidende Moment - auch die formale Struktur dieser Negation selbst signifikant, denn immerhin wirft sie die Frage auf, von welcher Qualität diese Erfahrung >tatsächlich` sein muss, wenn sie nur noch im Medium des Traums und im Verfahren der Negation, also in der Traumlogik, gemacht werden kann.

Im Begriff der Traumlogik konvergieren traum- und literaturtheoretische Voraussetzungen: In der Traumtheorie setzt die Traumlogik die Erzählbarkeit des Traums voraus; ${ }^{25}$ in der literarischen Analyse - hier bei Kleist und Kafka - setzt beispielsweise Klaus Jeziorkowski eine Dislozierung des Bekannten, eine "Verrückung und Entstellung « voraus. ${ }^{26}$ Dass der Traum darüber hinaus doch - aber eben in seiner narratologischen Funktionalisierung - zumindest an den psychoanalytisch inspirierten Topos der Wunscherfüllung (die allerdings nie in der Wirklichkeit, sondern eben immer nur im Erzählen glückt) erinnert, zeigt Franziska Mayer in ihrer 
Arbeit, die die Erzählstrategien generell mit »Wunscherfüllungen « (so der Titel) identifiziert. ${ }^{27}$ Der Traum ist daher nicht vom Wachsein oder von der Wirklichkeit, wie es in Schnitzlers Traumnovelle heißt, abgehoben. Der Traum ist die Negation des Wachseins, die Irrealisierung der Realität, aber zudem immer auch das Komplement - und unterliegt daher einer strengen Traumlogik. Marina Rauchenbacher hat in ihrer Untersuchung gezeigt, dass an den Raum- und Zeitstrukturen abgelesen werden kann, wie phantastische Sujets nicht nur erzeugt, sondern in Konfrontation gebracht werden mit »einem rational begründbaren und tradiertem (Geschichts-)Wissen, was sowohl Leo Perutz als auch Alexander Lernet-Holenia durch die wiederholte Verarbeitung historischer Stoffe demonstrieren $«{ }^{28}$ Kurz gesagt: Phantastisches und historisches Sujet sind nur die zwei Seiten ein und derselben Medaille in diesem Erzählmodell. Und ganz zu Recht kann Rüdiger Görner, mit Bezug auf Schnitzler, diese Novelle als Lernet-Holenias »Traumnovelle« bezeichnen..$^{29} \mathrm{Im}$ Traum werden nach der Logik des Komplements die Realitätsbedingungen überhaupt erst durchschaubar. Und in diesem Sinne - einer Poetologie des Erzählens bei Lernet-Holenia - ließen sich seine phantastischen Sujets als erzählerisch raffinierte Realitätsdiagnosen lesen. So lassen sich zwei Bezugsfelder benennen, die Lernet-Holenias Traumlogik besser zu konturieren helfen. Von dem einen Feld setzt er sich mit seiner Traumlogik eher ab - das sind die erwähnten Kriegsdarstellungen aus der Zwischenkriegszeit vor allem in der deutschen Literatur, auf der anderen Seite aber knüpft seine Traumlogik an andere Texte an, die aus dem Kontext Österreich-Ungarns stammen. Neben Schnitzler wäre hier insbesondere Franz Kafka, aber auch Franz Werfel, Stefan Zweig oder Joseph Roth zu nennen. Auch wenn es keine aussagekraftigen Rezeptionsdokumente zu der Frage gibt, ob Lernet-Holenia die Art reflektiert hat, wie Kafka »Traumproduktion und literarische Arbeit « ${ }^{30}$ eng zusammenrückt, kann Kafkas Modell zumindest poetologisch als musterbildend für Lernet-Holenia angesehen werden. Grundsätzlich mag man festhalten, dass der Traum in der Literatur des späten Habsburgerreiches ab 1900 und in der österreichischen Literatur danach eine besondere Rolle spielt. Ob das »Ineinander von Schein und Wirklichkeit [...] ein Bestandteil der österreichischen Daseinsform « sei, wie Peter Kampits feststellt, bleibe dahingestellt, aber seine Überzeugung, dass die österreichische Philosophie seit der Jahrhundertwende um 1900 
das Verhältnis von Schein und Wirklichkeit so hinterfragt, dass daraus eine Reihe von Schriftstellern und Schriftstellerinnen ihr poetologisches Schreibprinzip ableiten, was zudem noch durch historische Erfahrungen unterstützt wird, ist nicht von der Hand zu weisen. Der Traum spielt dabei als Narrationsform oder -technik eine bedeutende Rolle. ${ }^{31}$ Allerdings hat keiner der genannten Autoren jenes Traummodell, das Kafka oder Schnitzler vorbereitet hatten, so konzentriert als Erzählmodell für eine unsichere Identität verwendet wie Lernet-Holenia.

\section{7.}

Ähnliche Strukturen lassen sich im Roman Die Standarte finden, wenn auch abstrakter und komplexer gestaltet. Der Fähnrich Menis, auch er Ich-Erzähler einer Binnengeschichte, verliebt sich in Belgrad kurz vor dem Zusammenbruch der Armee durch das Auseinanderdriften der unterschiedlichen Nationalitäten in die Hofdame Resa. Er ist bereit, alles zu opfern, nur um Resa zu sehen und sie zu erobern. Er ist ein Draufgänger, dem militärische Disziplin nicht viel gilt. Als es ihm schließlich gelingt, Resas Liebe zu gewinnen, wird sie bedeutungslos, weil er - übrigens von einem Kameraden, der seine väterliche Herkunft nicht anzugeben weiß: hier wieder das biografische Element der unsicheren Identität - die Standarte seines Regiments übernimmt. Sie wird ihm zum Symbol für die Treue zu einem Staat und einem Reich, und zwar gerade in dem Moment, als sie sich in Auflösung befinden. Er muss phantastische Wege durchlaufen, z.B. in den unterirdischen Gängen der Belgrader Festung, dem Konak, die als Totenreich ebenso wie als Gebärmutter gedeutet werden können, so dass sein Weg und insbesondere sein Ausgang aus dem Höhlensystem sowohl Tod, aber auch Geburt und Wiedergeburt gleichermaßen ist. Danach bringt er die Standarte nach Wien zum Kaiser, der gerade Schönbrunn in Richtung Exil verlässt, während die Standarte verbrannt wird.

Die Patrouille Bagges und der unterirdische Gang Menis' führen beide Helden aus dem Krieg hinaus in ein Traumreich, in dem die wahren Verhältnisse auf den Kopf gestellt sind. Scheitern verwandelt sich in Erfolg, Tod in Leben, Abenteuer in Disziplin, Unsicherheit in Sicherheit - oder umgekehrt. So draufgängerisch sich der Held auch um sein Liebesglück bemüht, so bedeutungslos wird die Geliebte, als das Problem der unsiche- 
ren Identität durch die Erzählung eines geheimnisvollen Kameraden über einen anderen Kameraden offenkundig geworden ist. Das ist durchaus bemerkenswert, nicht nur, weil nunmehr dem selbst gegebenen militärischen Auftrag, die Standarte an den Ursprung des Kaiserreichs, zum Kaiser, zurückzubringen, höchste Priorität eingeräumt wurde, während Menis zuvor, um sein Liebesglück mit Resa zu erreichen, durchaus bereit gewesen ist, militärische Anordnungen mit List, Täuschung und Chupze zu übertreten; sondern auch, weil die Liebe als Modell wechselseitiger Anerkennung als Beglaubigung der unsicheren Identität denkbar gewesen wäre, aber ganz offenkundig nicht in Funktion tritt, obschon Resa dies durchaus andeutet.

Der Austausch des Objekts des Begehrens, die Substitution der Frau durch die Standarte, macht auf die Bedeutung des Krieges aufmerksam, denn die Standarte soll eine eindeutige militärische Funktion übernehmen, nämlich das Regiment (auch historisch) repräsentieren und seinen Zusammenhalt garantieren. Das eigentümliche Verhalten von Menis, die Frau gerade in dem Moment durch die Standarte als Objekt des Begehrens auszutauschen, als die Standarte funktionslos wird, entspricht allerdings dieser (Traum-)Logik des Krieges in der Erzählung. Was in der geschilderten Realität bedeutungslos geworden ist, gewinnt höchste Bedeutung im Erleben des Protagonisten. In Wien, nach etlichen Abenteuern und dem Verlust von Kameraden, kommt das böse Erwachen, nachdem die Standarten verbrannt sind (und noch im Feuer die heraufdämmernde Nazizeit als Vision sichtbar wird): „Dann fiel das Feuer wieder in sich zusammen, das Traumgesicht verging [...]. «2 $^{32}$ Die Ehe mit Resa ist dann nur noch eine reine Ehe aus Vernunft und Konvention, von Liebesheirat kann keine Rede mehr sein.

Ein letzter Aspekt sei noch angedeutet: Dass in beiden Fällen mit gerade fachmännischer Akribie die Umstände militärischer Reiterei geschildert werden, die in einem Zeitalter des Maschinengewehrs völlig wehrlos ist, ist nicht nur ein Hinweis auf den Anachronismus dieser Form von Kriegsführung, sondern mehr noch für die Extemporalität des damit ausgelösten Geschehens. Ja, gerade der Anachronismus des Pferdes und des Reitens ist direkt mit dem Gestus des Erzählens verbunden. Das Reiten ist das intradiegetische Korrelat zum - bezogen auf die Binnengeschichten - extradiegetischen Prozess des Erzählens. Reiten hier und Erzählen dort bedeuten eine Dynamisierung jener realen Ausgangsverhältnisse, die zu ihrer Irrealisierung im Traum und vor allem in der Traumerzählung führen. In dieser Hinsicht lässt sich dieses equestrische Motiv beispielsweise 
auch mit Hartmut Langes Roman Ulanenpatrouille (1940) oder mit Werner Bergengruens Roman Der letzte Rittmeister (1952) in Verbindung bringen.

8.

Tatsächlich aber gilt als Grundprinzip in beiden Texten, dass sich Eros in Thanatos verwandelt. Bagge wird beschuldigt, Frauen hätten sich seinetwegen umgebracht, Resa bedeutet Menis, als er sie schließlich ehelichen kann, nichts mehr, nachdem die Standarte verbrannt ist. Das alles habe ich mit dem Titel einer Traumlogik des Krieges bezeichnet. Was allerdings ist die Funktion dieser Traumlogik? Und was lässt sich aus dieser Funktionsbestimmung für ein Modell von >writing war ablesen? Antworten auf diese Fragen sollen noch kurz angedeutet werden.

Dass Eros sich in Thanatos in jenen Handlungsabschnitten verwandelt, in denen das Individuum eine Passage extremer, ja existenzieller Verunsicherung durchläuft (persönliche und familiäre Identität, ontologischer Status), das lässt sich auf ein Inventar romantischer Elemente zurückführen, was als Hinweis darauf gelten kann, dass die ästhetischen Prinzipien eines Modells von >writing war` weniger im Realismus einer Kriegsdarstellung gegeben sind, sondern vielmehr in epistemologischen und ontologischen Fragen einer Evaluation der geschilderten Erfahrungen. Lernet-Holenia zeigt mit solchen Traumlogiken die Exterritorialität und Extemporalität von Erfahrungen, die der Erste Weltkrieg gerade im Bereich der Habsburger Monarchie mit sich gebracht hat. Die Frage, wie man das Exterritoriale und das Extemporale, also dasjenige, was für die Figuren in der Rahmensituation in unwiederbringlicher Zeit und an unzugänglichen Orten geschehen ist, dennoch im Hier und Jetzt erzählen kann, beantwortet Lernet-Holenia mit dem Konzept einer impliziten Traumlogik, die darauf abzielt, mit dem Erzählen zugleich eine Vergegenwärtigung und Reterritorialisierung der erzählenden Figur zu leisten.

In der Standarte gibt es auch einen deutschen Offizier, Bottenlauben, eine Figur, die aufgrund ihres Unverständnisses die Funktion erfüllt, die im Text gelieferten Erklärungen der spezifisch österreichischen Verhältnisse zu motivieren. Tatsächlich scheint aber das Ensemble insgesamt auf eine Besonderheit der österreichischen Situation und ihrer literarischen Verarbeitung aufmerksam zu machen. Während deutsche Autoren ein Modell von > writing war $<$ zwischen Sachlichkeit und Expressionismus entwickeln, entwickelt Lernet-Holenia eine Traumlogik, die deutlich macht, dass es in seiner Literatur nicht um die Vergegenwärtigung des Krieges, sondern um 
eine Erfahrung geht, die ihre eigenen konstitutiven Koordinaten verloren hat; eine Kriegserfahrung, deren destruktive Drift vor der eigenen Selbstvergewisserung (in der Identität mit sich selbst) nicht mehr Halt macht. Der Traum wird zu einer Erfahrungsweise, in der die Erschütterung von Erfahrung selbst noch einmal thematisch werden kann, eine negative Epistemologie.

Bei Lernet-Holenia lässt sich also das Besondere dieser österreichischen Situation nachlesen: Man hatte nicht nur einen Krieg, man hatte seine Identität verloren. Ob man dabei schon von einem Beitrag zur österreichischen Identität in postimperialer Zeit sprechen will, bleibe dahingestellt. Robert Dassanowsky zumindest würdigt den Beitrag, den Lernet-Holenia mit seinen Texten zu dieser Frage geleistet hat, gerade dort, wo die eigene Identität unsicher geblieben ist. ${ }^{33}$ Lernet-Holenias Erzählwerk schreibt mit seinem Beitrag zu einer Kompensation und Aufarbeitung dieser Situation immerhin Literaturgeschichte.

\section{Literaturverzeichnis}

Alt, Peter-André: Der Schlaf der Vernunft. Literatur und Traum in der Literatur. München: Beck 2002.

Angelow, Jürgen (Hg.): Der Erste Weltkrieg auf dem Balkan. Perspektiven der Forschung. Berlin: Be.bra-Wiss.-Verl. 2011.

Dassanovsky, Robert: Phantom Empires: The Novels of Alexander Lernet-Holenia and the Question of Postimperial Austrian Identity. Riverside: Ariadne Press 1996.

Görner, Rüdiger: Nachwort. In: Alexander Lernet-Holenia: Der Baron Bagge. Novelle. Frankfurt/M., Leipzig: Insel 2001, S. 105-112.

Hüppauf, Bernd: Kriegsliteratur. In: Enzyklopädie Erster Weltkrieg. Hg. Gerhard Hirschfeld, Gerd Krumeich, Irina Renz. Erneut akt. u. erw. Studienausgabe. Paderborn: Schöningh 2014, S. 177-191.

Jeziorkowski, Klaus: Das Bett. In: Nach erneuter Lektüre. Franz Kafkas »Der Proceß«. Hg. Hans-Dieter Zimmermann. Würzburg: Königshausen \& Neumann 1992, S. 95-107.

Jeziorkowski, Klaus: Traum-Raum und Text-Höhle. Beobachtungen an dramatischen Szenen von Heinrich von Kleist. In: Ist mir getroumet min leben? Vom Träumen und vom Anderssein. Hg. Andre Schnyder. Göppingen: Kümmerle-Verl. 1998, S. 215-224.

Jünger, Ernst: In Stahlgewittern. Historisch-kritische Ausgabe. Hg. Helmuth Kiesel. Stuttgart: Klett-Cotta 2013.

Jünger, Ernst: Kriegstagebuch 1914-1918. Hg. Helmuth Kiesel. Stuttgart: Klett-Cotta 2010. Kampits, Peter: Zwischen Schein und Wirklichkeit. Österreichische Philosophie als Ausdruck eines ambivalenten Verhältnisses zur Realität. In: "Sein und Schein - Traum und Wirklichkeit«. Zur Poetik österreichischer Schriftsteller/innen im 20. Jahrhundert. Hgg. Herbert Arlt, Manfred Diersch. Frankfurt/M.: Lang 1994, S. 7-18. 
Kiesel, Helmuth: Geschichte der literarischen Moderne: Sprache, Ästhetik, Dichtung im zwanzigsten Jahrhundert. München: Beck 2004.

Kirschstein, Daniela: Writing War. Kriegsliteratur als Ethnographie bei Ernst Jünger, LouisFerdinand Céline und Curzio Malaparte. Würzburg: Königshausen \& Neumann 2014.

Kreutzer, Hans Joachim: Der Kleist-Preis 1912-1932-1985. Rede zu seiner Wiederbegründung. In: Kleist-Jahrbuch 1986. Stuttgart, Weimar: Metzler 1986, S. 11-18.

Lernet-Holenia, Alexander: Das lyrische Gesamtwerk. Hg. Roman Rocek. Wien: Zsolnay 1989.

Lernet-Holenia, Alexander: Der Baron Bagge. Novelle. Mit einem Nachwort von Rüdiger Görner. Frankfurt/M.: Insel 2001.

Lernet-Holenia, Alexander: Die Standarte. Roman. Mit einem Nachwort von Rüdiger Görner. Frankfurt/M.: Insel 2002.

Lernet-Holenia, Alexander: Fragmente aus verlorenen Sommern. Gedichte. Hg. u. mit einem Nachwort v. Rüdiger Görner. Wien: Zsolnay 2001.

Lüth, Reinhard: Drommetenrot und azurblau. Studien zur Affinität von Erzähltechnik und Phantastik in Romanen von Leo Perutz und Alexander Lernet-Holenia. Meitingen: Corian Verlag 1988.

Magris, Claudio: Der habsburgische Mythos in der österreichischen Literatur. Neuaufl. Wien: Zsolnay 2000.

Mayer, Franziska: Wunscherfüllungen. Erzählstrategien im Prosawerk Alexander LernetHolenias. Köln, Weimar, Wien: Böhlau 2005.

Rauchenbacher, Marina: Wege der Narration. Subjekt und Welt in den Texten von Leo Perutz und Alexander Lernet-Holenia. Wien: Praesens 2006.

Rauchensteiner, Manfried: Der Erste Weltkrieg und das Ende der Habsburgermonarchie 1914-1918. Wien: Böhlau 2014.

Rocek, Roman: Die neun Leben des Alexander Lernet-Holenia: Eine Biographie. Wien 1997.

Spiel, Hilde: Lernet-Holenia, Alexander. In: Neue österreichische Biografie. Bd. 20. Wien: Österreichische Akademie der Wissenschaften 1979, S. 88-94.

Spiel, Hilde: Welche Welt ist meine Welt? Erinnerungen 1946-1989. München: List 1990.

Strelka, Joseph: Zwischen Wirklichkeit und Traum. Das Wesen des Österreichischen in der Literatur. Tübingen: Francke 1994.

Türcke, Christoph: Philosophie des Traums. München: Beck 2011.

Werber, Niels; Kaufmann, Stefan; Koch, Lars (Hgg.): Erster Weltkrieg. Kulturwissenschaftliches Handbuch. Stuttgart, Weimar: Metzler 2014.

Werber, Niels: Die Geopolitik der Politik. Eine Vermessung der medialen Weltraumordnung. München: Beck 2007.

Werber, Niels: Geopolitik zur Einführung. Hamburg: Junius 2014.

Werber, Niels: Geopolitik. Vom >Platz an der Sonne zum >Volk ohne Raum‘. In: Erster Weltkrieg. Kulturwissenschaftliches Handbuch. Hgg. Niels Werber, Stefan Kaufmann, Lars Koch. Stuttgart,Weimar: Metzler 2014, S. 5-50. 
